\title{
Covid-19: Viral Pathogenesis and The Host Immune Response
}

\author{
Nasiru Usman Adabara ${ }^{1}$, Sherifat Ozavize Enejiyon ${ }^{1, *}$, Faruk Adamu Kuta ${ }^{1}$, \\ Ayanwale Oluwatobi Abraham $^{1}$ and Samia Alkhalil ${ }^{2}$ \\ ${ }^{1}$ Department of Microbiology, School of Life Sciences, Federal University of Technology Minna, Nigeria; ${ }^{2}$ Department of Clinical \\ Laboratory Sciences, College of Applied Medical Sciences, Shaqra University, Saudi Arabia.
}

Received: Sep 9, 2020; Revised: Dec 7, 2020; Accepted: January 12, 2021

\begin{abstract}
COVID-19, a pandemic caused by a betacoronavirus known as SARS-CoV-2 (severe acute respiratory syndrome coronavirus-2) has recorded 18354342 number of cases and 696147 deaths globally as of August 5, 2020. It was first recorded in Wuhan, China in 2019. The virus bears close resemblance to SARS-CoV-1 and MERS-CoV that have emerged and caused outbreaks of deadly human diseases. The main component of the virus responsible for the host range specific tropism and pathogenicity is the S-glycoprotein. The primary route of transmission of infection is through human to human via close contact, usually through spraying of droplets from sneeze or cough of an infected person. The incubation period for COVID-19 following viral infection is between 2 to 14 days. The target cells of SARS-CoV-2 are those cells that highly expressed ACE2 (angiotensin-converting enzyme 2). Viral receptor binds to the ACE2, to allow the virus entry into the cell via endosomal pathway. The host innate immune system detects the viral infection by using pattern recognition receptors which result in activation of downstream signalling cascade. Understanding the virulence factors contributing to pathology, host immune responses and strategies employed by the virus in bypassing host immune response is paramount in developing therapeutic options that can help to tackle the COVID-19 pandemics.
\end{abstract}

Keywords: COVID-19, coronavirus, receptors, immune response, pathogenesis.

\section{Introduction}

Corona Virus Disease 2019, abbreviated as COVID-19, is caused by a novel betacoronavirus, SARS-CoV-2 (severe acute respiratory syndrome coronavirus-2) (Kowalik et al., 2020). It is a newly emerging virus previously named 2019 novel coronavirus (2019-nCoV). The international committee on the taxonomy of viruses (ICTV) christened the new coronavirus as severe acute respiratory syndrome coronavirus 2 (SARS-CoV-2) on the $11^{\text {th }}$ day of February, 2020 due to its close genetic relatedness to SARS-CoV that caused an outbreak in 2002/2003 (Wu et al., 2020; CSG-ICT, 2020). An outbreak of the disease was first recorded in Wuhan, Hubei Province of China in December 2019, from which it disseminated to other parts of China and every other country. It was later declared a pandemic in March 2020 by the World Health Organization (Cucinotta and Vanelli, 2020). Currently, more than 6 million people have been infected globally with over 369, 258 deaths (WHO, 2020). The newly emerging virus, SARS-CoV-2 is an enveloped, un-segmented, positive sensed single stranded-RNA virus found within the subfamily "Coronavirinae", which includes viruses that infect humans and mammals. Three of these viruses known to cause human diseases have emerged during the last two decades and have caused widespread outbreaks of deadly human diseases. These highly pathogenic zoonotic coronaviruses include SARS
coronavirus-1(SARS-CoV-1) which was first observed in 2002, Middle East respiratory syndrome coronavirus (MERS-CoV) first identified in 2012 and SAR-CoV-2 which was first reported towards the end of 2019 (Dorsten et al., 2003; Zaki et al., 2012; Wu et al., 2020).

The clinical manifestation, morbidity rate, mortality rate and the case fatality ratio have slowly emerged. However, there are still some confusion about the pathogenesis and the specific role of the host immune responses in combating the infection. A lot of conclusion is being drawn from cumulated data on the previous coronaviruses namely SARS-CoV-1 and MERS-CoV because of their close genetic relatedness to the novel SARS-CoV-2. This review examines issues related to SARS-CoV-2 pathogenesis as well as the specific role played by the host immunity during infection drawing insights from other published works on the outbreak of COVID-19.

\section{Virulence Factors}

The occurrence and course of clinical manifestation in COVID-19 depend on pathogen factors which include virus type, virulence factors, viral load, mutation, replication and viability of the virus in vitro (Tang et al., 2020). Meanwhile, host factors such as the age of an infected person and the presence of comorbidities have also been identified to affect the clinical outcome of the viral infection (Abduljalil and Abduljalil, 2020).

\footnotetext{
* Corresponding author e-mail: enejiyon.sherifat@futminna.edu.ng.
} 
Coronaviruses are crown-like shaped, enveloped viruses of about $100 \mathrm{~nm}$ in diameter that are surrounded by spikes which protrude from the envelope. The virus genome is complemented by about 14 open reading frames (ORF). Each ORF encodes an array of proteins, accessory proteins, structural proteins and non-structural proteins that have important roles in virulence and propagation of the virus. The virus consists of four important structural proteins, membrane (M) glycoprotein, envelope (E) glycoprotein spike (S) glycoprotein and nucleocapsid (N) glycoprotein, as well as 16 nonstructural proteins (Fehr and Perlman, 2015; Kim et al., 2020).

Membrane and envelope glycoproteins are required for virus maturation, assembly, and replication. Together, they form the viral envelope as well as determine its shape. $\mathrm{N}$-glycoprotein forms an enclosure around the virus RNA genome known as viral nucleocapsid while spike glycoprotein is the elemental determinant of pathogenicity and host tropism (Hulswit et al., 2016; Felsenstein et al., 2020).

$\mathrm{S}$ glycoprotein is a transmembrane protein found in the outer region of the virus with a molecular weight of 150 $\mathrm{kDa}$. It enables the binding of viruses to host cells by forming homotrimers projecting on the surface of the virus to bind ACE2 found in cells of various organs including the kidney, liver and the lung (Fehr and Perlman, 2015). Furin-like protease of host cell split S protein into two subunits (S1 and S2). S1 subunit consists of two important domains; receptor binding domain (RBD) and N-terminal domain (NTD) (Wall et al., 2020). The RBD of the S glycoprotein has six critical amino acids that are responsible for binding to receptor ACE2 on host cells as well as the determination of virus cellular and host tropism (Andersen et al., 2020). On the other hand, the S2 functions in mediating the fusion between the virus and host cellular membrane (Guo et al., 2020).

Nonstructural proteins (nsps) carry out very essential roles in several processes in viruses as well as in the host cells; Nsp5 and Nsp3 encode proteases enzymes; chymotrypsin-like main protease and papain-like protease respectively. These enzymes help in the expression of cytokines, production and cleavage of viral amino acids polyprotein.

The virus demonstrates a high replication number (Anastassopoulou et al., 2020; Li et al., 2020) and it is more infectious probably as a result of acquisition of furincleavage site, which makes it spread very easily amongst people than the previous coronaviruses (Liu et al., 2020). The genome of SARS-CoV-2 consists of a sequence encoding amino acids PRRA and $\mathrm{R}$ within the original sequence of the $\mathrm{S}$ glycoprotein, generating a polybasic cleavage site (RRAR) for Furin protease at the junction of S1 and S2, leading to increase in the virus infectivity (Andersen et al., 2020; Liu et al., 2020). Combination of RBD and an insertion of cleavage site in genome of the virus have been reported to increase its infectivity in a study involving analysis of genomic sequences of SARSCoV-2 (Wu et al., 2020).

SAR-COV-2 has been indicated to evolve into leucine (L) and serine (S) types from the analyses of 103 SARS$\mathrm{CoV}-2$ genomes, where the serine type might be the ancestral type and leucine type might spread rapidly and be more aggressive (Tang et al., 2020). It has been suggested that variations in genome of SARS-CoV-2 might provide a basis for multiple sources outbreak (Zhang et al., 2020; Ceraolo and Giorgi, 2020).

\section{Viral Recognition by the Immune System}

Pathogenesis of coronavirus disease is still under research; the first site of infection with SARS-CoV-2 is airways epithelial cells. COVID-19 affects only the lungs in most patients as it is mainly a respiratory disease. The transmission of viral infection is primarily between humans through spraying droplets from cough or sneeze of an infected person in close contact with a susceptible person. The incubation period of COVID-19 is between 2 to 14 days, during which the virus can be spread (CDC, 2020). On the average, an infected person has the potential to infect at least two other people (Wu et al., 2020). Fever, cough, sore throat, fatigue and shortness of breath are the most common symptoms of the disease.

The functional receptor of SARS-CoV-2, angiotensin converting enzyme 2 (ACE2) plays a critical role in the pathogenesis of the virus. It provides the virus the opportunity of entry into human cells (Shereen et al., 2020; Lan et al., 2020). S glycoprotein of SARS-CoV-2 binds to ACE2. The binding domains of S-protein are present in 331 to 524 residues. Coding variants of ACE2 display high similarity in structural and binding affinity with the S glycoprotein of SARS-CoV-2. The cells of the host most commonly targeted by SARS-CoV-2 are those cells that highly expressed ACE2 and these include epithelial cells of oesophagus and ileum, alveolar cells, proximal tubule cells of kidney, myocardial cells and bladder urothelial cells (Chen et al., 2020; Zhou et al., 2020). Subsequent to the receptor binding, the virus gains entry into the cell through the endosomal pathway. The virus entry is mediated by $\mathrm{S}$ glycoprotein activated by the host cell type II transmembrane serine protease (TMPRSS2) present on the host cell surfaces (Ou et al., 2020). TMPRSS2 will clear the ACE2 and also activate S glycoproteins attached to the receptor by cleaving it into S1 and S2 subunit (Simmons et al., 2013; Rabi et al., 2020). S2 subunit facilitates the fusion of virus to the host cell membranes. Upon entry, the genomic material of the virus is released within the cytoplasm. The virus mRNA released into the cytoplasm is subjected to translation in the nuclei to produce replicase polyproteins (pp1a and pp1b) by ribosomal frame-shifting (Masters, 2006). The polyproteins are processed by protease enzymes encoded in Nsp3 and Nsp5. Subsequent cleavage of pp1a and pp1b into small proteins (Nsps1-Nsps11 and Nsps1-Nsps16 respectively) occurs. Viral RNA and nucleocapsid proteins interaction occur in the endoplasmic reticulum-Golgi complex compartment leading to the assemblage of the virion which can be liberated from the infected cell through exocytosis to infect adjacent cells (Hoffmann et al., 2020; Zhou et al., 2020).

\section{Immune Effector cells and their Activation}

With RNA viruses especially the coronaviruses, the human innate immune system uses the PRRs (pattern recognition receptors) such as TLR (toll-like receptors), RIG-I (retinoic acid inducible gene-I-like receptor), NLR (nucleotide-binding oligomerization domain like receptor), MDA5 (melanoma differentiation-associated protein 5 
receptor) and other free small receptor molecules located in the cytoplasm to recognize coronavirus PAMPs (pathogen associated molecular patterns) in order to detect the viral infection (Li et al., 2020; Kumar et al., 2020). PAMPs in the form of viral genomic RNA comprising nucleic acids, carbohydrate moieties, glycoproteins, lipoproteins and/or the intermediates products of viral replication (dsRNA) are recognized by the PRR. Each of the PRRs can then induce various biological responses through the activation of adapter proteins. TLRs results in the translocation of NF- $\mathrm{KB}$ and IRF3 while RIG-I and MDA5 effect in the activation of IRF3 (Yi et al., 2020; Li et al., 2020).

Recognition events by PRRs lead to the activation of the downstream signaling cascade using specific signal adapter proteins to produce immune system effector cells followed by their translocation into the nuclei. NF- $\mathrm{KB}$ and IRF3 (transcription factors) promote the synthesis of type I interferons (IFNs) and other pro-inflammatory cytokines in the nuclei (de Witt et al., 2016). Type I IFN via the cell surface receptor; IFN- $\alpha$ receptor complex (IFNAR) subsequently activates the Janus kinase-signal transducer and activator of transcription (JAK-STAT) signaling pathway, where JAK1 and JAK2 kinases phosphorylate STAT 1 and 2. STAT1/2 produce a complex with IRF9 and together, enter the nucleus to begin the transcription of interferon-stimulated genes, which leads to the suppression of viral replication by limiting viral spread and promotion of phagocytosis of viral particles by macrophages (Bergmann and Silvermann, 2020; Prompetchara et al., 2020). However, excess expression of pro-inflammatory cytokines and chemokines such as IFN- $\gamma$, IFN- $\alpha$, IL-33, IL-21, IL-18, IL-12, IL-6, IL-1 $\beta$, TGF $\beta$, TNF- $\alpha$ and MCP1 from effector cells of immune system, result in hyperinflammation which will ultimately lead to acute respiratory distress syndrome (ARDS) (Chen et al., 2010; Li et al., 2020).

$\mathrm{T}$ lymphocytes (CD4+) are promptly activated into $\mathrm{T}$ helper (Th1) cells when fragmented viral structural protein particles are presented by the major histocompatibility complex or human leukocyte antigen (HLA) and then recognized by virus-specific cytotoxic $\mathrm{T}$ lymphocytes. CD4+ $\mathrm{T}$ promotes the production of virus specific antibody by activating $\mathrm{T}$ cell-dependent $\mathrm{B}$ cells. Activated B cells will produce two specific immunoglobulins (Ig); IgM that last for only 12 weeks and IgG that can last for longer period and also provide long lasting immunity (Rabi et al., 2020; Astuti and Ysrafil, 2020). Activated Thelper cells are able to stimulate CD8+, which are part of the effectors of $\mathrm{T}$ lymphocyte that are cytotoxic and can destroy coronaviruses infected cells. Pro-inflammatory cytokines including interleukins- 12 and interferon- $\alpha$ are generated by T-helper cells via NF-kB signaling pathway. Generated cytokines induce inflammatory $\mathrm{T}$ cells, monocytes and neutrophils which induce inflammatory response (Zhou et al., 2020). These monocytes and T cells might move into blood circulation from where monocytes transformed into macrophages in tissues (Tang et al., 2020). Several cytokines including IL-6 in combination with dendritic cells (Nazinitsky and Rosenthal, 2010) trigger cytokine release syndrome (CRS) (Zhou et al., 2020). These responses allow the suppression of the viral infection inside the infected organs, clearance of the virus as well as the repair of the damaged tissues.

\section{Virus Response to Host Immune System}

Viral pathogenicity depends, in part, on the response of the virus against immune responses mounted by the host immune system. There are various strategies employed by coronaviruses in their response to the host immune system (Kikkert, 2020). Below are some of the specific mechanisms detected to play protective role in coronaviruses pathogenicity.

Viruses try to escape recognition by PRR in the cytosol through the formation of vesicle using some non-structural proteins by forming a capsule composed of protein and intracellular membrane around its genetic material. Viruses avoid recognition by MDA5 through the synthesis of RNA terminus crown to hide its 5' end thereby mimicking RNA terminus crown of human cells ( $\mathrm{Li}$ et al., 2020; Petrosillo et al., 2020; Siddique and Mehra, 2020).

Apart from avoiding recognition by PRR which is important for the initiation of host immune response, coronaviruses employ mechanisms that directly disrupt host defenses. Evidence of interruption of some processes of protein synthesis within cells infected with coronaviruses has been provided (Gaete-Argel et al., 2019). Viruses employ proteases to disrupt regulatory processes such as interferon activation within the infected cells that are dependent on ubiquitin. SARSCoV-2 has been found to cause the alteration of ubiquitination and the degradation of RIG-I/MDA5/MAVS/TRAF3/IRF3/IRF7 pathways (Siu et al., 2009). Important pathways for host specific responses against viruses such as RIG I and IRF3/7 can be targeted and blocked by viral proteins in various ways; inhibition of mitochondrial antiviral signaling (MAVS) proteins synthesis, increase in stimulation of pro-inflammatory cytokines, NF-kB and necroptosis leading to inadequate synthesis of type 1 interferon and impairment of specific immune responses which will invariably result in hyper-inflammation, enhanced cellular death and cytokine storm ( $\mathrm{Li}$ et al., 2020; Yi et al., 2020).

SARS-CoV-2 viral proteins target and inhibit various signaling proteins involved in the activation of TLRs/TRIF/MyD88/IkB/NF-kB/MAPK/AP-1 pathway thereby causing inhibition of the synthesis of proinflammatory cytokines (Conti et al., 2020). Nonstructural proteins are involved in blockage and inhibition of interferon pathway and NF- $\mathrm{KB}$ pathway while interferoninducible mRNA nuclear export complex can be impeded by Orf6 (Dai et al., 2020; Gordon et al., 2020).

Coronavirus can combine with non-neutralizing antibodies to form immune complexes which often lead to a severe inflammation (Hohdatsu et al., 1998; Jaume et al., 2011). These complexes can bind to the Fc receptor of specific neutralizing antibodies thereby enabling SARS$\mathrm{CoV}-2$ to penetrate and multiply in target cells such as antigen-presenting cells and phagocytes. Maffia et al. (2019) reported that SARS-CoV-2 directly infects macrophages and $\mathrm{T}$ cells by attaching to CD209 receptor on macrophages thereby invading effector cells in vascular and cardiac tissues.

Impairment of protective immune responses by SARS$\mathrm{CoV}-2$ allows it to easily propagate and cause severe damage to infected cells in organs such as intestine and kidney where ACE2 are highly expressed. Proinflammatory cells such as granulocytes and macrophages 
in the damaged cells induce inflammatory response in the lung (Shi et al., 2020).

\section{Conclusion}

COVID-19 caused by SARS-CoV-2 is still a pandemic affecting the lives of millions of people worldwide. SARS$\mathrm{CoV}-2$ propagates and replicate easily in target cells by actively disrupting host immune responses. It induces hyper-inflammation which often leads to cytokine storm or life threatening condition by recruiting uninfected cells to the focal point of infection and/or infecting effector cells of immune system.. Understanding the virulence factors contributing to pathology, host immune responses and strategies employed by the virus in bypassing host immune response is paramount in developing therapeutic options that can help to tackle the COVID-19 pandemics.

\section{References}

Abduljalil JM and Abduljalil BM. 2020. Epidemiology, genome, and clinical features of the pandemic SARS-CoV-2: a recent review. New Microbe and New Infect., 35(100672): 1-8.

Anastassopoulou C, Russo L, Tsakris A and Siettos C. 2020. Data-based analysis, modelling and forecasting of the novel coronavirus (2019- nCoV) outbreak. PLoS One., 15(3): e0230405.

Andersen KG, Rambaut A, Lipkin WI, Holmes EC and Garry RF. 2020. The proximal origin of SARS-CoV2. Nat Med., 26: 450452.

Astuti I and Ysrafil. 2020. Severe Acute Respiratory Syndrome Coronavirus 2 (SARSCoV-2): An overview of viral structure and host response. Diabetes Metab Syndr., 14(4): 407-412.

Bergmann CC and Silvermann RH. 2020. COVID-19: Coronavirus replication, pathogenesis, and therapeutic strategies. Cleve Clin J Med., 87(6): 321-327.

Center for Disease Control and Prevention (2020). "Symptoms of Novel Coronavirus (2019-nCoV)". Https://www.cdc.gov/coronavirus/2019ncov/about/symptoms.html (May 24, 2020).

Ceraolo C and Giorgi FM. 2020. Genomic variance of the 2019$\mathrm{nCoV}$

coronavirus. J Med Virol., 92(5): 522-528.

Chen L, Li X, Chen M, Feng Y and Xiong C. 2020. The ACE2 expression in human heart indicates new potential mechanism of heart injury among patients infected with SARS-CoV-2. Cardiovas Res., 116(6): 1097-1100.

Conti P, Ronconi G, Caraffa A, Gallenga C, Ross R, Frydas I et al. 2020. Induction of pro-inflammatory cytokines (IL-1 and IL6) and lung inflammation by Coronavirus-19 (COVI-19 or SARSCoV-2): anti-inflammatory strategies. J Biol Regul Homeost Agents., 34(2): 327-331.

Coronaviridae Study Group of the International Committee on Taxonomy (CSG-ICT) of Virus. 2020. The species severe acute respiratory syndrome-related coronavirus: classifying 2019-nCoV and naming it SARS-CoV-2. Nat Microbiol., 5(4): 536-544.

Cucinotta D and Vanelli M. 2020. WHO declares COVID-19 a pandemic. Acta Biomed., 91(1): 157-160.

Dai W, Zhang B, Jiang XM, Su H, Li J, Zhao Y et al. 2020. Structure-based design of antiviral drug candidates targeting the SARS-CoV-2 main protease. Science., 368(6497): 1331-1335.

de Wit E, van Doremalen N, Falzarano D and Munster VJ. 2016. SARS and MERS: recent insights into emerging coronaviruses. Nat Rev Microbiol., 14(8): 523-534.
Drosten C, Gunther S, Preiser W, van der Werf S, Brodt HR, Becker S et al. 2003. Identification of a novel coronavirus in patients with severe acute respiratory

syndrome. N Engl J Med., 348(20): 1967-1976.

Fehr AR and Perlman S. 2015. Coronaviruses: an overview of their replication and pathogenesis. Method Mol Biol., 1282: 1-23.

Felsenstein S, Herbert JA, McNamara PS and Hedrich CM. 2020. COVID-19: immunology and treatment options. Clin Immunol., 215: 108448.

Gaete-Argel A, Márquez CL, Barriga GP, Soto-Rifo R and Valiente-Echeverria F. 2019. Strategies for success. Viral infections and membraneless organelles. Front Cell Infect Microbiol., 9: 336.

Gordon DE, Jang GM, Bouhaddou M, Xu J, Obernier K, White $\mathrm{KM}$ et al. 2020. A SARS-CoV-2 protein interaction map reveals targets for drug repurposing. Nature, 583(7816): 459-468.

Guo YR, Cao QD, Hong ZS, Tan YY, Chen SD, Jin HJ et al. 2020. The origin, transmission and clinical therapies on coronavirus disease 2019 (COVID-19) outbreak-an update on the status. Mil Med Res., 7(1): 11.

Hoffmann M, Kleine-Weber $\mathrm{H}$ and Pohlmann S. 2020. A multibaic cleavage site in the spike protein of SARS-CoV-2 is essential for infection of human lung cells. Mol cell., 78(4): 779784.

Hohdatsu T, Yamada M, Tominaga R, Makino K, Kida K and Koyama H. 1998. Antibody-dependent enhancement of feline infectious peritonitis virus infection in feline alveolar macrophages and human monocyte cell line U937 by serum of cats experimentally or naturally infected with feline coronavirus. $J$ Vet Med Sci., 60(1): 49-55.

Hulswit RJ, de Haan CA and Bosch BJ. 2016. Coronavirus Spike Protein and Tropism Changes. Adv Virus Res., 96: 29-57.

Jaume M, Yip MS, Cheung CY, Leung HL, Li PH, Kien F et al. 2011. Anti-severe acute respiratory syndrome coronavirus spike antibodies trigger infection of human immune cells via a $\mathrm{pH}$ - and cysteine protease-independent FcyR pathway. J Virol., 85(20): 10582-10597.

Kikkert M. 2020. Innate immune evasion by human respiratory RNA viruses. J Innate Immunol., 12(1): 4-20.

Kim YI, Kim SG, Kim SM, Kim EH, Park SJ, Yu KM et al. 2020. Infection and rapid transmission of SARS-CoV-2 in Ferrets. Cell Host Microbe., 27(5): 704-709.e2.

Kowalik MM, Trzonkowski P, Łasińska-Kowara M, Mital A, Smiatacz T and Jaguszewski M. 2020. COVID-19 - Toward a comprehensive understanding of the disease. Cardio J., 27(2): 99114.

Kumar S, Nyodu R, Maurya VK and Saxena SK. 2020. Morphology, genome organization, replication, and pathogenesis of severe acute respiratory syndrome coronavirus 2 (SARS-CoV2). Saxena SK (Eds.), Coronavirus Disease 2019 (COVID-19), Medical Virology: from Pathogenesis to Disease Control.

Lan J, Ge J, Yu J, Shan S, Zhou H, Fan S et al. 2020. Structure of the SARS-CoV-2 spike receptor binding domain bound to the ACE2 receptor. Nature., 581(7808): 215-220.

Li G, Fan Y, Lai Y, Han T, Li Z, Zhou P et al. 2020. Coronavirus infections and immune responses. J Med Virol., 92(4): 424-432.

Li Q, Guan X, Wu P, Wang X, Zhou L, Tong Y et al. 2020. Early transmission dynamics in Wuhan, China, of novel coronavirusinfected pneumonia. New Engl J Med., 382(13): 1199-1207.

Liu H, Wu C, Yang Y, Liu Y, Zhang P, Wang Y et al. 2020. Furin, a potential therapeutic target for COVID-19. ChinaXiv. 
Maffia P and Guzik TJ. 2019. When, where, and how to target vascular inflammation in the post-CANTOS era?. Eur Heart J., 40(30): 2492-2494.

Masters PS. 2006. The molecular biology of coronaviruses. Adv Virus Res., 6: 193-292.

Nazinitsky A and Rosenthal KS. 2010. Cytokine storms: systemic disasters of infectious diseases. Infect Dis Clin Pract., 18(3): 188192.

Ou X, Liu Y, Lei X, Li P, Mi D, Ren L et al. 2020. Characterization of spike glycoprotein of SARS-CoV immune cross-reactivity with SARS-CoV. Nat Commun., 11(1): 1620.

Petrosillo N, Viceconte G, Ergonul O, Ippolito G and Petersen E. 2020. COVID-19, SARS and MERS: are they closely related? Clin Microbiol Infect., 26(6): 729-734.

Prompetchara E, Ketloy C and Palaga T. 2020. Immune responses in COVID-19 and potential vaccines: Lessons learned from SARS and MERS epidemic. Asian Pac J Allergy Immunol., 38(1): 1-9.

Rabi FA, Al Zoubi MS, Kasasbeh GA, Salameh DM and AlNasser AD. 2020. SARS-CoV-2 and coronavirus disease 2019: what we know so far. Pathogen., 9(3): 231-235.

Shereen MA, Khan S, Kazmi A, Bashir N and Siddique R. 2020. COVID-19 infection: origin, transmission, and characteristics of human coronaviruses. J Adv Res., 24: 91-98.

Shi Y, Wang Y, Shao C, Huang J, Gan J, Huang X et al. 2020. COVID-19 infection: the perspective on immune responses. Cell Death Differ., 27(5): 1451-54.

Siddiqi HK and Mehra MR. 2020. COVID-19 illness in native and immunosuppressed states: A clinical-therapeutic staging proposal. J Heart Lung Transplant., 39(5): 405-407.

Simmons G, Zmora P, Gierer S, Heurich A and Pöhlmann S 2013. Proteolytic activation of the SARS coronavirus spike protein: cutting enzymes at the cutting edge of antiviral research. Antiviral Res., 100(3): 605-614.

Siu KL, Kok KH, Ng MH, Poon VKM, Yuen KY, Zheng BJ et al. 2009. Severe acute respiratory syndrome coronavirus $M$ protein inhibits type I interferon production by impeding the formation of TRAF3.TANK.TBK1/IKKepsilon complex. J Biol Chem., 284(24): 16202-16209
Tang X, Wu C, Li X, Song Y, Yao X, Wu X et al. 2020. On the origin and continuing evolution of SARS-CoV-2. Natl Sci Rev., nwaa036.

Walls AC, Park YJ, Tortorici MA, Wall A, McGuire AT and Veesler D. 2020. Structure, function, and antigenicity of the SARS-CoV-2 spike glycoprotein. Cell., 181(2): 281-292.

World Health Organization (WHO) (2020). "Naming the coronavirus disease (COVID-19) and the virus that causes it". https://www.who.int/emergencies/diseases/novel-coronavirus2019/technical-guidance/naming-the-coronavirus-disease-(covid2019)-and-the-virus-that-causes-it (May 24, 2020).

Wu A, Niu P, Wang L, Zhou H, Zhao X, Wang W et al. 2020. Mutations, recombination and insertion in the evolution of 2019nCoV. ChinaXiv.

Wu F, Zhao S, Yu B, Chen YM, Wang W, Song ZG et al. 2020. A new coronavirus associated with human respiratory disease in China. Nature., 579(7798): 265-269.

Yi Y, Lagniton PNP, Ye S, Li E and Xu RH. 2020. COVID-19: what has been learned and to be learned about the novel coronavirus disease. Int J Biol Sci., 16(10): 1753-1766.

Zaki M, van Boheemen S, Bestebroer TM, Osterhaus AD and Fouchier RA. 2012. Isolation of a novel coronavirus from a man with pneumonia in Saudi Arabia. N Engl J Med., 367(19): 1814-1820.

Zhang L, Yang JR, Zhang Z and Lin Z. 2020. Genomic variations of SARSCoV-2 suggest multiple outbreak sources of transmission. MedRxiv., PPR: PPR114841.

Zhou G, Chen S and Chen S. 2020. Advances in COVID-19: the virus, the pathogenesis, and evidence-based control and therapeutic strategies. Front Med., 14(2): 117-125.

Zhou Y, Fu B, Zheng X, Wang D, Zhao C, Qi Y et al. 2020. Aberrant pathogenic GM-CSF+ $\mathrm{T}$ cells and inflammatory CD14+CD16+ monocytes in severe pulmonary syndrome patients of a new coronavirus. bioRxiv. 